DO-TH 2000/15

hep-ph/0011300

November 2000

\title{
On the Determination of the Polarized Sea Distributions of the Nucleon
}

\author{
M. Glück, A. Hartl, and E. Reya \\ Universität Dortmund, Institut für Physik, \\ D-44221 Dortmund, Germany
}

\begin{abstract}
The possibilities to determine the flavor structure of the polarized sea (antiquark) distributions of the nucleon via vector boson $\left(\gamma^{*}, W^{ \pm}, Z^{0}\right)$ production at high energy polarized hadron-hadron colliders, such as the Relativistic Heavy-Ion Collider (RHIC), are studied in detail. In particular the perturbative stability of the expected asymmetries in two representative models for the (un)broken flavor structure are investigated by confronting perturbative QCD leading order predictions of the expected asymmetries with their next-to-leading order counterparts.
\end{abstract}




\section{Introduction}

The determination of the polarized parton content of the nucleon via measurements of the inclusive structure functions $g_{1}^{p, n}\left(x, Q^{2}\right)$ does not provide detailed information concerning the flavor structure of these distributions, in complete analogy to unpolarized deep inelastic structure functions. In particular the flavor structure of the antiquark (sea) distributions is not fixed and one needs to resort to semi-inclusive deep inelastic hadron production for this purpose [1, 2]. The resulting antiquark distributions $\Delta \bar{q}$ are, however, not reliably determined by this method [3, [- for the time being due to their dependence on the rather poorly known quark fragmentation functions at low scales.

A more reliable determination is provided via inclusive vector boson $\left(\gamma^{*}, W^{ \pm}, Z^{0}\right)$ production in polarized hadron-hadron collisions as envisaged at RHIC (BNL) or at HERA-N (DESY) whose potential for the determination of $\Delta \bar{u}$ and $\Delta \bar{d}$ will be studied in this paper in leading (LO) as well as next-to-leading (NLO) order of QCD.

Recently the LO Drell-Yan $\left(\gamma^{*}\right)$ dilepton production process for future polarized $\overrightarrow{p p}$ and $\vec{p} \vec{d}$ collision experiments has been suggested and studied [5, 6] for delineating the flavor-asymmetry of the polarized light sea distributions $\Delta \bar{u}\left(x, Q^{2}\right) \neq \Delta \bar{d}\left(x, Q^{2}\right)$. In particular, the relativistic field theoretic chiral quark-soliton model [7, 8, 9, 2] as well as a more recent analysis based on the statistical parton model [10] predict $\left|\Delta \bar{d}\left(x, Q^{2}\right)\right|>$ $\Delta \bar{u}\left(x, Q^{2}\right)$. Similar expectations [11, 17, 12, 10, 13], partly from first principles, hold also for the unpolarized light sea distributions, $\bar{d}>\bar{u}$, which have been already confirmed, as is well known, by Drell-Yan $\mu^{+} \mu^{-}$production [14] $p p$ and $p d$ experiments [15]. In general, the flavor-asymmetry of the light sea distributions can be understood in terms of flavor mass asymmetries and 'Pauli-blocking' effects being related to the Pauli exclusion principle [16, 17, 13].

In order to analyze the determination of (flavor-asymmetric) light antiquark (sea) 
distributions one needs some alternative models for $\Delta \bar{q}$, introduced in Section 2 , and the possibility of their experimental distinction will be investigated in Section 3. Our conclusions are drawn in Sect. 4 and the Appendix contains all expressions of LO and NLO cross sections relevant for our calculations.

\section{Models for the Polarized Antiquark Distributions of the Nucleon}

(i) Standard unbroken sea scenario: Here one assumes, as in most analyses of polarization data performed thus far, a flavor symmetric sea, i.e.

$$
\Delta \bar{u}\left(x, Q^{2}\right)=\Delta \bar{d}\left(x, Q^{2}\right)=\Delta \bar{s}\left(x, Q^{2}\right) \equiv \Delta \bar{q}\left(x, Q^{2}\right)
$$

where as usual $\Delta u_{\text {sea }}=\Delta \bar{u}, \Delta d_{\text {sea }}=\Delta \bar{d}$ and $\Delta s=\Delta \bar{s}$. The adopted LO and NLO distributions will be taken from the recent analysis of the AA Collaboration [18], in particular their LO and NLO-1 ones.

(ii) Broken sea scenario: Here one assumes $\Delta \bar{u}\left(x, Q^{2}\right) \neq \Delta \bar{d}\left(x, Q^{2}\right) \neq \Delta \bar{s}\left(x, Q^{2}\right)$, i.e. a broken flavor symmetry as motivated by the situation in the corresponding unpolarized sector 15, 13]. As mentioned in the Introduction, present polarization data do not provide detailed and reliable information concerning flavor symmetry breaking and we shall therefore utilize antiquark distributions extracted via the phenomenological ansatz of [17] which is confirmed in the unpolarized sector and which moreover agrees well with the predictions obtained within the framework of the chiral quark-soliton model [7, 8, 9, 2] and with a recent analysis of semi-inclusive deep inelastic data [4].

These flavor-asymmetric distributions, henceforth denoted by $\Delta f^{\prime}$, are related to the (flavor-symmetric) AAC 18 distributions $\Delta f$ by the relations 


$$
\begin{aligned}
\Delta u^{\prime}\left(x, Q_{0}^{2}\right) & =\Delta u\left(x, Q_{0}^{2}\right)-\phi(x) \\
\Delta \bar{u}^{\prime}\left(x, Q_{0}^{2}\right) & =\Delta \bar{q}\left(x, Q_{0}^{2}\right)+\phi(x) \\
\Delta d^{\prime}\left(x, Q_{0}^{2}\right) & =\Delta d\left(x, Q_{0}^{2}\right)+\phi(x) \\
\Delta \bar{d}^{\prime}\left(x, Q_{0}^{2}\right) & =\Delta \bar{q}\left(x, Q_{0}^{2}\right)-\phi(x) \\
\Delta u_{v}^{\prime}\left(x, Q_{0}^{2}\right) & =\Delta u_{v}\left(x, Q_{0}^{2}\right)-2 \phi(x) \\
\Delta d_{v}^{\prime}\left(x, Q_{0}^{2}\right) & =\Delta d_{v}\left(x, Q_{0}^{2}\right)+2 \phi(x) \\
\Delta s^{\prime}\left(x, Q_{0}^{2}\right) & =\Delta \bar{s}^{\prime}\left(x, Q_{0}^{2}\right)=\Delta s\left(x, Q_{0}^{2}\right)=\Delta \bar{s}\left(x, Q_{0}^{2}\right)=\Delta \bar{q}\left(x, Q_{0}^{2}\right) \\
\Delta g^{\prime}\left(x, Q_{0}^{2}\right) & =\Delta g\left(x, Q_{0}^{2}\right)
\end{aligned}
$$

with $\Delta q_{v} \equiv \Delta q-\Delta \bar{q}$ and $\Delta \bar{q}$ given by (2.1) at the input scale $Q_{0}^{2}=1 \mathrm{GeV}^{2}$, and where

$$
\phi=-\Delta \bar{q} \frac{\Delta u-\Delta d}{\Delta u+\Delta d-2 \Delta \bar{q}}
$$

as follows from the Pauli-blocking relation [17]

$$
\Delta \bar{d}^{\prime}\left(x, Q_{0}^{2}\right) / \Delta \bar{u}^{\prime}\left(x, Q_{0}^{2}\right)=\Delta u^{\prime}\left(x, Q_{0}^{2}\right) / \Delta d^{\prime}\left(x, Q_{0}^{2}\right)
$$

combined with the constraints $(q=u, d)$

$$
\begin{aligned}
\Delta q^{\prime}\left(x, Q_{0}^{2}\right)+\Delta \bar{q}^{\prime}\left(x, Q_{0}^{2}\right) & =\Delta q\left(x, Q_{0}^{2}\right)+\Delta \bar{q}\left(x, Q_{0}^{2}\right) \\
\sum_{q=u, d} \Delta q_{v}^{\prime}\left(x, Q_{0}^{2}\right) & =\sum_{q=u, d} \Delta q_{v}\left(x, Q_{0}^{2}\right)
\end{aligned}
$$

needed [19] to preserve the quality of the fit [18] to $g_{1}^{p, n}\left(x, Q^{2}\right)$ within the standard unbroken sea scenario. Note that $\Delta \bar{u}^{\prime}-\Delta \bar{d}^{\prime}=2 \phi$ and that the 'breaking' function $\phi(x)$ in (2.3) can be simply parametrized in LO and NLO as well,

$$
\begin{aligned}
x \phi(x)_{\mathrm{LO}} & =x^{0.536}\left(0.27-0.14 x+13.57 x^{2}-10.17 x^{3}\right)(1-x)^{10.5} \\
x \phi(x)_{\mathrm{NLO}} & =x^{0.32}\left(0.12+0.90 x-6.68 x^{2}+20.06 x^{3}-20.36 x^{4}\right)(1-x)^{7.7},
\end{aligned}
$$

which allows to form Mellin $n$-moments of $\phi$ needed for performing the $Q^{2}$-evolutions in $n$-moment space as described, for example, in [20]. The resulting distributions are shown 
in fig. 1 at the input scale $Q_{0}^{2}$ as well as at $Q^{2}=25 \mathrm{GeV}^{2}$ and $M_{W}^{2}$, and for comparison we also show the flavor-symmetric sea density $\Delta \bar{q}$ in (2.1) of AAC [18]. It should be emphasized that our broken distributions should not be used well below $x \simeq 10^{-2}$ since our breaking-ansatz [17, 19] $\Delta \bar{d}^{\prime} / \Delta \bar{u}^{\prime} \equiv(\Delta \bar{q}-\phi) /(\Delta \bar{q}+\phi)=(\Delta u-\phi) /(\Delta d+\phi)$ which results in (2.3) gives rise to artificial oscillations [19] below $x=0.01$ due to the appearance of differences of parton distributions in (2.3). All our subsequent analyses employ these broken distributions well above $x=10^{-2}$. (For obvious reasons we skip from now on the 'prime' notation in the above equations for the flavor-asymmetric distributions and simply refer to 'AAC' in connection with the original $\mathrm{AAC}$-densities with their flavor-symmetric sea $\Delta \bar{q}$.

Furthermore, the unpolarized cross sections needed for the asymmetry calculations of Sect. 3 will be evaluated with the LO and NLO unpolarized parton distributions GRV98 21] utilized by the AAC [18.

\section{Cross Section Asymmetries for Inclusive Hadronic Vector Boson Production}

As stated in the Introduction, the inclusive hadronic vector boson production yields reliable information on the antiquark (sea) content of the nucleon. We shall consider double (single) asymmetries as obtained with doubly (singly) polarized hadron beams. The relevant expressions for the corresponding cross sections in LO and NLO are collected, for convenience, in the Appendix.

We shall successively study $\gamma^{*}$ and $W^{ \pm}, Z^{0}$ production in Sections 3.1 and 3.2, respectively, starting with estimates of expected production rates at RHIC (BNL) and turning then to a study of the optimal ways to gain insight about the flavor structure of the antiquark distributions in the nucleon. 


\section{$3.1 \gamma^{*}$ Production}

The expected production rates at RHIC are determined via the following expected energies and integrated luminosities [22]: $\sqrt{S}=50-500 \mathrm{GeV}, \mathcal{L}_{\vec{p} \vec{p}}(\sqrt{S}) \simeq(\sqrt{S} / 500 \mathrm{GeV})$ $800 p b^{-1}$. The polarization rate for the proton beams is expected to be $P_{\vec{p}} \simeq 0.7$ and will be obviously lower for polarized neutrons, i.e. deuteron beams for example. In our estimates for the expected statistical errors we shall use $P=0.7$ everywhere which represents an idealized underestimate of these rather crucial errors for the case of $d(n)$ beams.

Asymmetries involving only polarized protons, although easiest to be measured experimentally, are unlikely to provide unique signatures for a flavor-broken polarized light sea:

$$
\begin{aligned}
A_{\vec{p} \vec{p}}^{\gamma^{*}} & \equiv \sigma_{\vec{p} \vec{p}}^{\gamma^{*}} / \sigma_{p p}^{\gamma^{*}} \\
& \stackrel{\mathrm{LO}}{\simeq}-\frac{4[\Delta u(1) \Delta \bar{u}(2)+\Delta \bar{u}(1) \Delta u(2)]+\Delta d(1) \Delta \bar{d}(2)+\Delta \bar{d}(1) \Delta d(2)}{4[u(1) \bar{u}(2)+\bar{u}(1) u(2)]+d(1) \bar{d}(2)+\bar{d}(1) d(2)}
\end{aligned}
$$

where $(\Delta) \sigma$ denotes the relevant differential cross sections summarized in the Appendix and where we make use of the simple abbreviated ' 1,2 ' notation introduced in the Appendix as well. The size and even the sign of this asymmetry depends already strongly on the choice of the particular set of unbroken polarized parton densities [23]. For our sets of (un)broken distributions in Sect. 2 we obtain, for example, at $\sqrt{S}=50 \mathrm{GeV}$ and for an invariant dilepton mass $M=5 \mathrm{GeV}$ in $\vec{p} \vec{p} \rightarrow \gamma^{*}(M) X \rightarrow \mu^{+} \mu^{-} X$ an asymmetry $A_{\vec{p} \vec{p}}^{\gamma^{*}} \simeq+2 \%$ for the unbroken (AAC) densities and $A_{\vec{p} \vec{p}}^{\gamma^{*}} \simeq-(4$ to 6$) \%$ for the flavor-broken scenario for all values of $x_{F}$.

As in the case of flavor-broken unpolarized parton distributions [14, 15], additional polarized $p n(p d)$ reactions are required for delineating the flavor structure of the polarized 
light (anti)quark sea $\Delta \bar{u}$ and $\Delta \bar{d}$ :

$$
\begin{aligned}
& A_{\vec{p} \pm \vec{n}}^{\gamma^{*}} \equiv \frac{\Delta \sigma_{\vec{p} \vec{p}}^{\gamma^{*}} \pm \Delta \sigma_{\vec{p} \vec{n}}^{\gamma^{*}}}{\sigma_{p p}^{\gamma^{*}}+\sigma_{p n}^{\gamma^{*}}} \\
& \stackrel{\mathrm{LO}}{\simeq}-\frac{[4 \Delta u(1) \pm \Delta d(1)][\Delta \bar{u}(2) \pm \Delta \bar{d}(2)]+[4 \Delta \bar{u}(1) \pm \Delta \bar{d}(1)][\Delta u(2) \pm \Delta d(2)]}{[4 u(1)+d(1)][\bar{u}(2)+\bar{d}(2)]+[4 \bar{u}(1)+\bar{d}(1)][u(2)+d(2)]} \\
& \stackrel{x_{F} \rightarrow 1}{\longrightarrow}-\frac{[4 \Delta u(1) \pm \Delta d(1)][\Delta \bar{u}(2) \pm \Delta \bar{d}(2)]}{[4 u(1)+d(1)][\bar{u}(2)+\bar{d}(2)]} \\
& R_{p \pm n} \equiv \frac{1}{2}\left(1 \pm \Delta \sigma_{\vec{p} \vec{n}}^{\gamma^{*}} / \Delta \sigma_{\vec{p} \vec{p}}^{\gamma^{*}}\right) \\
& \stackrel{\mathrm{LO}}{\simeq} \frac{1}{2} \frac{[4 \Delta u(1) \pm \Delta d(1)][\Delta \bar{u}(2) \pm \Delta \bar{d}(2)]+[4 \Delta \bar{u}(1) \pm \Delta \bar{d}(1)][\Delta u(2) \pm \Delta d(2)]}{4[\Delta u(1) \Delta \bar{u}(2)+\Delta \bar{u}(1) \Delta u(2)]+\Delta d(1) \Delta \bar{d}(2)+\Delta \bar{d}(1) \Delta d(2)} \\
& \stackrel{x_{F} \rightarrow 1}{\longrightarrow} \frac{1}{2} \frac{[4 \Delta u(1) \pm \Delta d(1)][\Delta \bar{u}(2) \pm \Delta \bar{d}(2)]}{4 \Delta u(1) \Delta \bar{u}(2)+\Delta d(1) \Delta \bar{d}(2)} \simeq \frac{1}{2}\left[1 \pm \frac{\Delta \bar{d}(2)}{\Delta \bar{u}(2)}\right]
\end{aligned}
$$

where for $x_{F} \rightarrow 1$, i.e. $x_{1} \rightarrow 1$ and $x_{2} \rightarrow 0$, the small $\Delta \bar{q}(1)$ terms can be neglected and in the very last 'crude' (LO) approximation of $R_{p \pm n}$ the $\Delta d(1)$ terms are neglected with respect to $4 \Delta u(1)$. This latter ratio of polarized cross sections in (3.3) has been suggested and studied in LO originally in [5] as a very sensitive observable for testing the flavor-asymmetry of the polarized light sea. Indeed, at small $x, R_{p \pm n}$ is sensitive directly to the ratio $\Delta \bar{d} / \Delta \bar{u}$ whereas $A_{\vec{p} \pm \vec{n}}^{\gamma^{*}}$ in (3.2) is proportional to the isoscalar and isovector combinations $\Delta \bar{u} \pm \Delta \bar{d}$.

First we present in fig. 2 the unpolarized cross section $d \sigma_{p p}^{\gamma^{*}} / d M d x_{F}$ for $\sqrt{S}=50$ and $100 \mathrm{GeV}$. It is obvious that only for $\left|x_{F}\right| \lesssim 0.5$ and not too large dilepton masses $M$ useful production rates can be obtained. From

$$
x_{1,2}^{0}=\frac{1}{2}\left(\sqrt{x_{F}^{2}+4 M^{2} / S} \pm x_{F}\right)
$$

one infers that the small- $x_{2}$ region, relevant for our study of sea (antiquark) distributions, implies small $\tau \equiv M^{2} / S$ values. Here, furthermore, the NLO contributions are genuine 
$\mathcal{O}\left(\alpha_{s}\right)$ corrections and small. Since the relevant asymmetries decrease with $\sqrt{S}$ we have chosen $\sqrt{S}=50 \mathrm{GeV}$ and $M=5-8 \mathrm{GeV}$ to be the appropriate range for our study of asymmetries related to Drell-Yan dilepton pairs notwithstanding the fact, shown in fig. 2, that the unpolarized cross sections do increase with $\sqrt{S}$.

As expected, $A_{\vec{p}-\vec{n}}^{\gamma^{*}}$ and in particular $R_{p d} \equiv R_{p+n}$ are the best indicators for the flavor structure of the antiquark (sea) distibutions: Fig. 3 presents our LO and NLO results with the latter ones being, furthermore, remarkably stable with respect to sizeable variations of the factorization scale $\mu_{F}$. It should be noted that for all scenarios of polarized parton distributions with a flavor-symmetric light sea, $\Delta \bar{u}=\Delta \bar{d}$, we have $R_{p d} \rightarrow 1$ and $A_{\vec{p}-\vec{n}} \rightarrow 0$ as $x_{F} \rightarrow 1-$ a limit which is already reached for $x_{F} \gtrsim 0.2$ for most sets of polarized parton densities as illustrated for AAC in fig. 3. The statistical errors shown in fig. 3 are obtained from (see, e.g., 22, 24)

$$
\begin{aligned}
\Delta A_{\vec{h}_{1} \vec{h}_{2}} & \simeq \pm \frac{1}{P_{1} P_{2}} \frac{1}{\sqrt{4 \mathcal{L} \sigma_{h_{1} h_{2}}}} \\
\Delta R_{p \pm n} & \simeq \pm \frac{1}{2 P^{2}} \frac{1}{\sqrt{\left(A_{\vec{p} \vec{p}}\right)^{2}}} \frac{1}{\sqrt{4 \mathcal{L} \sigma_{p p}}}
\end{aligned}
$$

assuming $P_{1}=P_{2}=P=0.7$ for the beam polarizations, $\mathcal{L}=80 p b^{-1}$ and bin widths $\Delta x_{F}=0.1, \Delta M=1 \mathrm{GeV}$ for calculating bin-integrated cross sections.

\section{2 $W^{ \pm}$and $Z^{0}$ Production}

The production of these vector bosons via (un)polarized $p p(n)$ collisions, $p p(n) \rightarrow$ $W^{ \pm} X \rightarrow \mu^{ \pm} \stackrel{(-)}{\nu}_{\mu} X$ and $p p(n) \rightarrow Z^{0} X \rightarrow \mu^{+} \mu^{-} X$, affords of course higher c.m. energies which are, however, available at RHIC $\left(\sqrt{S}_{\max }=500 \mathrm{GeV}\right)$ [22]. The expected cross sections, presented in fig. 4, are comparable to those for $\gamma^{*}$ production at $\sqrt{S}=50$ $\mathrm{GeV}$ in fig. 2. These cross sections are partly more sensitive to the flavor structure of the light sea, although at far larger scales $\mu_{F} \sim M_{W^{ \pm}, Z^{0}}$, and may discern its polarized flavor 
structure not only in doubly polarized collisions but also in the single polarization mode [25]-27] where only one beam is polarized. Possible benefits of this latter mode are the expected lower statistical errors

$$
\Delta A_{\vec{h}_{1} h_{2}} \simeq \pm \frac{1}{P_{1}} \frac{1}{\sqrt{4 \mathcal{L} \sigma_{h_{1} h_{2}}}}
$$

i.e., a factor $P_{1}^{-1}$, as compared to $\left(P_{1} P_{2}\right)^{-1}$ for the doubly polarized mode in $(3.5)$, as well as possibly higher luminosities. As seen in fig. 4, the relevant rapidity range is $|y| \lesssim 1$ which covers the interesting range of Bjorken $-x$ for the sea distributions, $0.06 \lesssim x \lesssim 0.4$.

The relevant single and double helicity asymmetries are

$$
\begin{aligned}
& A_{\vec{p} p}^{W^{+}} \equiv \Delta \sigma_{\vec{p} p}^{W^{+}} / \sigma_{p p}^{W^{+}} \\
& \stackrel{\mathrm{LO}}{\simeq} \frac{-\Delta u(1) \bar{d}(2)+\Delta \bar{d}(1) u(2)}{u(1) \bar{d}(2)+\bar{d}(1) u(2)} \longrightarrow\left\{\begin{aligned}
-\Delta u(1) / u(1), & y \gtrsim+\frac{1}{2} \\
\Delta \bar{d}(1) / \bar{d}(1), & y \lesssim-\frac{1}{2}
\end{aligned}\right. \\
& A_{\vec{p} p}^{W^{-}} \equiv \Delta \sigma_{\vec{p} p}^{W^{-}} / \sigma_{p p}^{W^{-}} \\
& \stackrel{\mathrm{LO}}{\simeq} \frac{-\Delta d(1) \bar{u}(2)+\Delta \bar{u}(1) d(2)}{d(1) \bar{u}(2)+\bar{u}(1) d(2)} \longrightarrow\left\{\begin{aligned}
-\Delta d(1) / d(1), & y \gtrsim+\frac{1}{2} \\
\Delta \bar{u}(1) / \bar{u}(1), & y \lesssim-\frac{1}{2}
\end{aligned}\right. \\
& A_{\vec{p} \vec{p}}^{W^{+}} \equiv \Delta \sigma_{\vec{p} \vec{p}}^{W^{+}} / \sigma_{p p}^{W^{+}} \\
& \stackrel{\mathrm{LO}}{\simeq}-\frac{\Delta u(1) \Delta \bar{d}(2)+\Delta \bar{d}(1) \Delta u(2)}{u(1) \bar{d}(2)+\bar{d}(1) u(2)} \quad \stackrel{y \gtrsim+\frac{1}{2}}{\longrightarrow} \quad-\frac{\Delta u(1)}{u(1)} \frac{\Delta \bar{d}(2)}{\bar{d}(2)} \\
& A_{\vec{p} \vec{p}}^{W^{-}} \equiv \Delta \sigma_{\vec{p} \vec{p}}^{W^{-}} / \sigma_{p p}^{W^{-}} \\
& \stackrel{\mathrm{LO}}{\simeq}-\frac{\Delta d(1) \Delta \bar{u}(2)+\Delta \bar{u}(1) \Delta d(2)}{d(1) \bar{u}(2)+\bar{u}(1) d(2)} \quad \stackrel{y \gtrsim+\frac{1}{2}}{\longrightarrow} \quad-\frac{\Delta d(1)}{d(1)} \frac{\Delta \bar{u}(2)}{\bar{u}(2)}
\end{aligned}
$$

with obvious generalizations to $A_{\vec{p} d}^{W^{ \pm}}$and $A_{\vec{p} \vec{d}}^{W^{ \pm}}$, and where

$$
x_{1,2}^{0}=\left(M_{W}^{2} / S\right)^{\frac{1}{2}} e^{ \pm y} .
$$


In addition we also study the following ratios of singly and doubly polarized cross sections

$$
\begin{array}{ccc}
\frac{\Delta \sigma_{\vec{p} p}^{W^{+}}}{\Delta \sigma_{\vec{p} p}^{W^{-}}} \stackrel{\mathrm{LO}}{\simeq} \frac{-\Delta u(1) \bar{d}(2)+\Delta \bar{d}(1) u(2)}{-\Delta d(1) \bar{u}(2)+\Delta \bar{u}(1) d(2)} & \stackrel{y \lesssim-\frac{1}{2}}{\longrightarrow} & \frac{\Delta \bar{d}(1)}{\Delta \bar{u}(1)} \frac{u(2)}{d(2)} \\
\frac{\Delta \sigma_{\vec{p} d}^{W^{+}}}{\Delta \sigma_{\vec{p} d}^{W^{-}}} \stackrel{\mathrm{LO}}{\simeq} \frac{-\Delta u(1)[\bar{u}(2)+\bar{d}(2)]+\Delta \bar{d}(1)[u(2)+d(2)]}{-\Delta d(1)[\bar{u}(2)+\bar{d}(2)]+\Delta \bar{u}(1)[u(2)+d(2)]} \stackrel{y \lesssim-\frac{1}{2}}{\longrightarrow} \frac{\Delta \bar{d}(1)}{\Delta \bar{u}(1)} \\
\frac{\Delta \sigma_{\vec{p} \vec{p}}^{W^{+}}}{\Delta \sigma_{\vec{p} \vec{p}}^{W^{-}}} \stackrel{\mathrm{LO}}{\simeq} \frac{\Delta u(1) \Delta \bar{d}(2)+\Delta \bar{d}(1) \Delta u(2)}{\Delta d(1) \Delta \bar{u}(2)+\Delta \bar{u}(1) \Delta d(2)} \\
\frac{\Delta \sigma_{\vec{p} \vec{d}}^{W^{+}}}{\Delta \sigma_{\vec{p} \vec{d}}^{W-}} \stackrel{\mathrm{LO}}{\simeq} \frac{\Delta u(1)[\Delta \bar{u}(2)+\Delta \bar{d}(2)]+\Delta \bar{d}(1)[\Delta u(2)+d(2)]}{\Delta d(1)[\Delta \bar{u}(2)+\Delta \bar{d}(2)]+\Delta \bar{u}(1)[\Delta u(2)+\Delta d(2)]} \stackrel{\frac{y \lesssim-\frac{1}{2}}{\longrightarrow}}{\stackrel{\Delta u(1)}{\longrightarrow}} \underset{\frac{\Delta d(1)}{\Delta \bar{d}(1)}}{\frac{\Delta \bar{u}(2)}{\Delta \bar{u}(1)}}
\end{array}
$$

The singly polarized asymmetries in (3.8) and (3.9) are, for negative values of $y$, dominated by the polarized antiquark distributions. (Note that $y=-1$ corresponds to $x_{2}^{0} \simeq 0.06$.) The limiting values $\Delta q / q$ and $\Delta \bar{q} / \bar{q}$ of $A_{\vec{p} p}^{W^{ \pm}}$at the scale $\mu_{F}^{2}=M_{W}^{2}$ are shown in fig. 5 where they are compared with the flavor-unbroken (AAC) scenario as well. It is conceivable that such differences can be delineated by future RHIC experiments taken into account their expected statistical accuracy [22].

In fig. 6 we present the expected double spin asymmetries $A_{\vec{p} \vec{p}}^{W^{ \pm}}$as defined in (3.10) and (3.11) in the broken and unbroken (AAC) sea scenarios, as specified in fig. 1, in LO as well as in NLO of perturbative QCD. Here, at large scales $\mu_{F} \sim M_{W}$, the perturbative stability with respect to sizeable variations of the factorization scale $\mu_{F}$ is even more pronounced than for the Drell-Yan $\left(\gamma^{*}\right)$ asymmetries in fig. 3. The expected statistical errors which are reduced as compared to their size in the Drell-Yan $\left(\gamma^{*}\right)$ production asymmetries are shown in fig. 3. It is seen that in any case, LO or NLO estimates, a distinction between both scenarios is possible due to the sizeable reduction in the statistical errors involved which is mainly due to the increased luminosity at $\sqrt{S}=500 \mathrm{GeV}$ as compared to $\sqrt{S}=50 \mathrm{GeV}$, relevant for Drell-Yan $\left(\gamma^{*}\right)$ production as discussed in Section 3.1. We 
recall here the envisaged integrated luminosities at RHIC, i.e. $\mathcal{L}(\sqrt{S})=(\sqrt{S} / 500 \mathrm{GeV})$ $800 p b^{-1}$ and eq. (3.5). In fig. 7 we present the corresponding single asymmetries $A_{\vec{p} p}^{W^{ \pm}}$ of eqs. (3.8) and (3.9) which turn out be less sensitive to the flavor-broken sea densities than the double spin asymmetries at $y>0$ in fig. 6. An interesting feature demonstrated here is the quality of the 'crude' approximations in eqs. (3.8) and (3.9) which are well satisfied at $|y| \gtrsim \frac{1}{2}$ in the LO calculations where they are relevant. The ratios of singly and doubly polarized cross sections in eqs. (3.14) and (3.15), together with their limiting LO 'crude' approximations, are shown in fig. 8. Again, on account of the rather small expected statistical errors, it is likely that future measurements can discriminate between the flavor-broken (solid curves) and unbroken (dashed curves) light-sea scenarios.

Finally we present the following ratio of combinations of polarized and unpolarized cross sections [26], which turn out to be less sensitive to absolute normalization uncertainties,

$$
\begin{aligned}
a_{\vec{p} N}^{W} & \equiv \frac{\Delta \sigma_{\vec{p} p}^{W^{+}}+\Delta \sigma_{\vec{p} p}^{W^{-}}-\left(\Delta \sigma_{\vec{p} n}^{W^{+}}+\Delta \sigma_{\vec{p} n}^{W^{-}}\right)}{\sigma_{p p}^{W^{+}}+\sigma_{p p}^{W^{-}}+\sigma_{p n}^{W^{+}}+\sigma_{p n}^{W^{-}}} \\
& \stackrel{\mathrm{LO}}{\simeq} \frac{[\Delta u(1)-\Delta d(1)][\bar{u}(2)-\bar{d}(2)]-[\Delta \bar{u}(1)-\Delta \bar{d}(1)][u(2)-d(2)]}{[u(1)+d(1)][\bar{u}(2)+\bar{d}(2)]+[\bar{u}(1)+\bar{d}(1)][u(2)+d(2)]}
\end{aligned}
$$

and its corresponding doubly polarized counterpart

$$
\begin{aligned}
a_{\vec{p} \vec{N}}^{W} & \equiv \frac{\Delta \sigma_{\vec{p} \vec{p}}^{W^{+}}+\Delta \sigma_{\vec{p} \vec{p}}^{W^{-}}-\left(\Delta \sigma_{\vec{p} \vec{n}}^{W^{+}}+\Delta \sigma_{\vec{p} \vec{n}}^{W^{-}}\right)}{\sigma_{p p}^{W^{+}}+\sigma_{p p}^{W^{-}}+\sigma_{p n}^{W^{+}}+\sigma_{p n}^{W^{-}}} \\
& \stackrel{\mathrm{LO}}{\simeq} \frac{[\Delta u(1)-\Delta d(1)][\Delta \bar{u}(2)-\Delta \bar{d}(2)]-[\Delta \bar{u}(1)-\Delta \bar{d}(1)][\Delta u(2)-\Delta d(2)]}{[u(1)+d(1)][\bar{u}(2)+\bar{d}(2)]+[\bar{u}(1)+\bar{d}(1)][u(2)+d(2)]} .
\end{aligned}
$$

Both asymmetries have no polarized gluon contribution proportional to $\Delta g$ in NLO, which cancels in the various differences $\Delta \sigma_{\vec{p} p}-\Delta \sigma_{\vec{p} n}$ and $\Delta \sigma_{\vec{p} \vec{p}}-\Delta \sigma_{\vec{p} \vec{n}}$. Furthermore, the double helicity asymmetry in (3.18) vanishes for $\Delta \bar{u}=\Delta \bar{d}$ in LO as well as in NLO and is thus an interesting combination to observe the effects of a flavor-broken sea due to $\Delta \bar{u} \neq \Delta \bar{d}$. 
This is explicitly demonstrated in fig. 9 where the sizeably different expectations of the flavor-broken sea scenario should be easily discernible experimentally.

Finally, for $Z^{0}$ production we have found that the following two double and single spin asymmetries are best suited for the investigation of the flavor structure of the sea:

$$
\begin{aligned}
& A_{\vec{p} \vec{p}}^{Z^{0}} \equiv \Delta \sigma_{\vec{p} \vec{p}}^{Z^{0}} / \sigma_{p p}^{Z^{0}} \\
& \stackrel{\mathrm{LO}}{\simeq}-\frac{\alpha_{u}[\Delta u(1) \Delta \bar{u}(2)+\Delta \bar{u}(1) \Delta u(2)]+\alpha_{d}[\Delta d(1) \Delta \bar{d}(2)+\Delta \bar{d}(1) \Delta d(2)]}{\alpha_{u}[u(1) \bar{u}(2)+\bar{u}(1) u(2)]+\alpha_{d}[d(1) \bar{d}(2)+\bar{d}(1) d(2)]} \\
& A_{\vec{p}, p-n}^{Z^{0}} \equiv \frac{\Delta \sigma_{\vec{p} p}^{Z^{0}}-\Delta \sigma_{\vec{p} n}^{Z^{0}}}{\sigma_{p p}^{Z^{0}}+\sigma_{p n}^{Z^{0}}} \\
& \stackrel{\mathrm{LO}}{\simeq} \frac{-\left[\beta_{u} \Delta u(1)-\beta_{d} \Delta d(1)\right][\bar{u}(2)-\bar{d}(2)]-\left[\beta_{u} \Delta \bar{u}(1)-\beta_{d} \Delta \bar{d}(1)\right][u(2)-d(2)]}{\left[\alpha_{u} u(1)+\alpha_{d} d(1)\right][\bar{u}(2)+\bar{d}(2)]+\left[\alpha_{u} \bar{u}(1)+\alpha_{d} \bar{d}(1)\right][u(2)+d(2)]}
\end{aligned}
$$

where $\alpha_{q} \equiv v_{q}^{2}+a_{q}^{2}$ and $\beta_{q} \equiv 2 v_{q} a_{q}$ as explained and given in the Appendix. These asymmetries are depicted, together with their expected statistical errors, in fig. 10. It should be emphasized that these $Z^{0}$-production asymmetries are up to an order of magnitude larger in the flavor-broken $(\Delta \bar{u} \neq \Delta \bar{d})$ scenario than for flavor-symmetric sea densities $(\Delta \bar{u}=\Delta \bar{d})$ where they become almost unmeasurably small.

\section{Summary and Conclusions}

The possibility to determine the flavor structure of the polarized antiquark (sea) distributions of the nucleon via vector boson $\left(\gamma^{*}, W^{ \pm}, Z^{0}\right)$ production at high energy polarized hadron-hadron $(\vec{p} \vec{p}, \vec{p} \vec{n}(\vec{d}))$ colliders was investigated. The perturbative stability of the expected asymmetries for two representative models for the flavor structure of the sea distributions was studied and has shown that the predicted distinctive signatures for both flavor-symmetric and flavor-asymmetric models remain essentially unchanged in LO and 
NLO of perturbative QCD. This demonstrates that these characteristic and distinctive features are genuine signatures of the models under consideration for the flavor structure of the polarized sea.

In particular the polarized Drell-Yan $\left(\gamma^{*}\right)$ dilepton production asymmetry $A_{\vec{p}-\vec{n}}^{\gamma^{*}}$ in (3.2), as obtained from $\vec{p} \vec{p}$ and $\vec{p} \vec{n}$ collisions, or the ratio $R_{p d}$ of polarized $\vec{p} \vec{p}$ and $\vec{p} \vec{n}$ production cross sections in (3.3) provide us with characteristic and direct signatures for a flavor-broken polarized sea, $\Delta \bar{u} \neq \Delta \bar{d}$, as illustrated in fig. 3. At much larger factorization scales $\mu_{F}^{2} \sim M_{W, Z}^{2}$, the double spin asymmetries $A_{\vec{p} \vec{p}}^{W^{ \pm}}$for $W^{ \pm}$production in (3.10) and (3.11), or combinations of doubly polarized $\vec{p} \vec{p}$ and $\vec{p} \vec{n} W^{ \pm}$-production cross sections in (3.18) constitute similar clean and distinctive observables for studying the flavor structure of the polarized light sea, as shown in figs. 6 and 9. Somewhat less sensitive signatures for a flavor-broken sea are provided by the single spin asymmetries $A_{\vec{p} p}^{W^{ \pm}}$in (3.8) and (3.9), although they may give access directly to $\Delta \bar{d}\left(x, M_{W}^{2}\right)$ and $\Delta \bar{u}\left(x, M_{W}^{2}\right)$ in specific kinematic regions $(y \lesssim-0.5)$. Such direct signatures for $\Delta \bar{d}$ and $\Delta \bar{u}$ could also be obtained from studying ratios of $W^{+}$and $W^{-}$cross sections of singly and doubly polarized $p d$ collisions, cf. (3.14) and (3.16). Finally, the double spin asymmetry $A_{\vec{p} \vec{p}}^{Z^{0}}$ production at RHIC is an equally useful observable for delineating the flavor structure of the polarized sea, since it is expected to be about an order of magnitude larger for a flavor-broken $(\Delta \bar{u} \neq \Delta \bar{d})$ than for a flavor-symmetric $(\Delta \bar{u}=\Delta \bar{d})$ polarized sea scenario as shown in fig. 10 .

The resolution power of the asymmetries studied depends of course on the expected statistical errors which were estimated for the envisaged beam polarizations and luminosities at RHIC. They point towards the superiority of vector boson $\left(W^{ \pm}, Z^{0}\right)$ production (figs. 6 -10) over the common Drell-Yan $\gamma^{*}$ production (fig. 3) as a tool for studying the flavor structure of the polarized sea distributions. This derives mainly from the increased luminosity at the corresponding higher energies involved in vector boson production, i.e. $\sqrt{S} \simeq 500 \mathrm{GeV}$, as compared to $\sqrt{S} \simeq 50 \mathrm{GeV}$ relevant for $\gamma^{*}$ (dilepton) production as discussed in Section 3.1. 


\section{Appendix}

Here we summarize all those unpolarized and polarized cross sections for (Drell-Yan) vector boson $\left(\gamma^{*}, W^{ \pm}, Z^{0}\right)$ production in $\mathrm{LO}$ and $\mathrm{NLO}(\overline{\mathrm{MS}})$ needed for calculating the various spin-asymmetries suggested and studied in this paper.

In terms of cross sections of definite positive and negative hadron helicities $( \pm)$, an unpolarized cross section is generally defined by $\sigma=\frac{1}{4}\left(\sigma_{++}+\sigma_{+-}+\sigma_{-+}+\sigma_{--}\right)$. The relevant differential unpolarized Drell-Yan cross section for $h_{1} h_{2} \rightarrow \gamma^{*} X \rightarrow l^{+} l^{-} X$ can be written as

$$
\begin{aligned}
M^{2} \frac{\mathrm{d}{\sigma_{h} h_{2}}_{\gamma^{*}}\left(x_{F}, M^{2}, \mu_{F}^{2}\right)}{\mathrm{d} M^{2} \mathrm{~d} x_{F}}= & N^{\gamma^{*}} \sum_{q=u, d, s} e_{q}^{2} \int_{x_{1}^{0}}^{1} \mathrm{~d} x_{1} \int_{x_{2}^{0}}^{1} \mathrm{~d} x_{2} \\
\times & \left\{\left[D_{q \bar{q}}^{(0)}\left(x_{1}, x_{2}, x_{1}^{0}, x_{2}^{0}\right)+\frac{\alpha_{s}}{2 \pi} D_{q \bar{q}}^{(1)}\left(x_{1}, x_{2}, x_{1}^{0}, x_{2}^{0}, \frac{M^{2}}{\mu_{F}^{2}}\right)\right]\right. \\
& \times\left\{q\left(x_{1}, \mu_{F}^{2}\right) \bar{q}\left(x_{2}, \mu_{F}^{2}\right)+\bar{q}\left(x_{1}, \mu_{F}^{2}\right) q\left(x_{2}, \mu_{F}^{2}\right)\right\} \\
+ & \frac{\alpha_{s}}{2 \pi} D_{g q}^{(1)}\left(x_{1}, x_{2}, x_{1}^{0}, x_{2}^{0}, \frac{M^{2}}{\mu_{F}^{2}}\right) g\left(x_{1}, \mu_{F}^{2}\right)\left\{q\left(x_{2}, \mu_{F}^{2}\right)+\bar{q}\left(x_{2}, \mu_{F}^{2}\right)\right\} \\
& \left.+\frac{\alpha_{s}}{2 \pi} D_{q g}^{(1)}\left(x_{1}, x_{2}, x_{1}^{0}, x_{2}^{0}, \frac{M^{2}}{\mu_{F}^{2}}\right)\left\{q\left(x_{1}, \mu_{F}^{2}\right)+\bar{q}\left(x_{1}, \mu_{F}^{2}\right)\right\} g\left(x_{2}, \mu_{F}^{2}\right)\right\}
\end{aligned}
$$

with $N^{\gamma^{*}}=4 \pi \alpha^{2} / 9 S, \alpha_{s}=\alpha_{s}\left(\mu_{F}^{2}\right), D_{q \bar{q}}^{(0)}\left(x_{1}, x_{2}, x_{1}^{0}, x_{2}^{0}\right)=\delta\left(x_{1}-x_{1}^{0}\right) \delta\left(x_{2}-x_{2}^{0}\right) /\left(x_{1}^{0}+x_{2}^{0}\right)$ and according to the $\mathrm{NLO}(\overline{\mathrm{MS}})$ results of $[28,23]$

$$
\begin{aligned}
D_{q \bar{q}}^{(1)}\left(x_{1}, x_{2}, x_{1}^{0}, x_{2}^{0}, \frac{M^{2}}{\mu_{F}^{2}}\right)=C_{F} & \left\{\frac { \delta ( x _ { 1 } - x _ { 1 } ^ { 0 } ) \delta ( x _ { 2 } - x _ { 2 } ^ { 0 } ) } { x _ { 1 } ^ { 0 } + x _ { 2 } ^ { 0 } } \left[\frac{\pi^{2}}{3}-8+2 \operatorname{Li}_{2}\left(x_{1}^{0}\right)+2 \operatorname{Li}_{2}\left(x_{2}^{0}\right)\right.\right. \\
+ & \left.\ln ^{2}\left(1-x_{1}^{0}\right)+\ln ^{2}\left(1-x_{2}^{0}\right)+2 \ln \frac{x_{1}^{0}}{1-x_{1}^{0}} \ln \frac{x_{2}^{0}}{1-x_{2}^{0}}\right]
\end{aligned}
$$




$$
\begin{aligned}
& +\left(\frac { \delta ( x _ { 1 } - x _ { 1 } ^ { 0 } ) } { x _ { 1 } ^ { 0 } + x _ { 2 } ^ { 0 } } \left[\frac{1}{x_{2}}-\frac{x_{2}^{0}}{x_{2}^{2}}-\frac{x_{2}^{0}{ }^{2}+x_{2}^{2}}{x_{2}^{2}\left(x_{2}-x_{2}^{0}\right)} \ln \frac{x_{2}^{0}}{x_{2}}\right.\right. \\
& +\frac{x_{2}^{0}{ }^{2}+x_{2}^{2}}{x_{2}^{2}}\left(\frac{\ln \left(1-x_{2}^{0} / x_{2}\right)}{x_{2}-x_{2}^{0}}\right)_{+}+\frac{x_{2}^{0}{ }^{2}+x_{2}^{2}}{x_{2}^{2}} \frac{1}{\left(x_{2}-x_{2}^{0}\right)_{+}} \\
& \left.\left.\ln \frac{\left(x_{1}^{0}+x_{2}^{0}\right)\left(1-x_{1}^{0}\right)}{x_{1}^{0}\left(x_{1}^{0}+x_{2}\right)}\right]+(1 \leftrightarrow 2)\right) \\
& +\frac{G^{A}\left(x_{1}, x_{2}, x_{1}^{0}, x_{2}^{0}\right)}{\left[\left(x_{1}-x_{1}^{0}\right)\left(x_{2}-x_{2}^{0}\right)\right]_{+}}+H^{A}\left(x_{1}, x_{2}, x_{1}^{0}, x_{2}^{0}\right) \\
& +\ln \frac{M^{2}}{\mu_{F}^{2}}\left\{\frac{\delta\left(x_{1}-x_{1}^{0}\right) \delta\left(x_{2}-x_{2}^{0}\right)}{x_{1}^{0}+x_{2}^{0}}\left[3+2 \ln \frac{1-x_{1}^{0}}{x_{1}^{0}}+2 \ln \frac{1-x_{2}^{0}}{x_{2}^{0}}\right]\right. \\
& \left.\left.+\left(\frac{\delta\left(x_{1}-x_{1}^{0}\right)}{x_{1}^{0}+x_{2}^{0}} \frac{x_{2}^{0}{ }^{2}+x_{2}^{2}}{x_{2}^{2}} \frac{1}{\left(x_{2}-x_{2}^{0}\right)_{+}}+(1 \leftrightarrow 2)\right)\right\}\right\} \\
& D_{q g}^{(1)}\left(x_{1}, x_{2}, x_{1}^{0}, x_{2}^{0}, \frac{M^{2}}{\mu_{F}^{2}}\right)=T_{F}\left\{\frac { \delta ( x _ { 2 } - x _ { 2 } ^ { 0 } ) } { ( x _ { 1 } ^ { 0 } + x _ { 2 } ^ { 0 } ) x _ { 1 } ^ { 3 } } \left[\left(x_{1}^{0^{2}}+\left(x_{1}-x_{1}^{0}\right)^{2}\right) \ln \frac{\left(x_{1}^{0}+x_{2}^{0}\right)\left(1-x_{2}^{0}\right)\left(x_{1}-x_{1}^{0}\right)}{x_{1}^{0} x_{2}^{0}\left(x_{1}+x_{2}^{0}\right)}\right.\right. \\
& \left.+2 x_{1}^{0}\left(x_{1}-x_{1}^{0}\right)\right]+\frac{G^{C}\left(x_{1}, x_{2}, x_{1}^{0}, x_{2}^{0}\right)}{\left(x_{2}-x_{2}^{0}\right)_{+}}+H^{C}\left(x_{1}, x_{2}, x_{1}^{0}, x_{2}^{0}\right) \\
& \left.+\ln \frac{M^{2}}{\mu_{F}^{2}}\left\{\frac{\delta\left(x_{2}-x_{2}^{0}\right)}{\left(x_{1}^{0}+x_{2}^{0}\right) x_{1}^{3}}\left(x_{1}^{0^{2}}+\left(x_{1}-x_{1}^{0}\right)^{2}\right)\right\}\right\}
\end{aligned}
$$

where $C_{F}=4 / 3, T_{F}=1 / 2, \operatorname{Li}_{2}(x)=-\int_{0}^{x} \mathrm{~d} t \ln (1-t) / t$ and

$$
\begin{aligned}
G^{A}\left(x_{1}, x_{2}, x_{1}^{0}, x_{2}^{0}\right) & =\frac{\left(x_{1}+x_{2}\right)\left(x_{1}^{0}{ }^{2} x_{2}^{02}+x_{1}^{2} x_{2}^{2}\right)}{x_{1}^{2} x_{2}^{2}\left(x_{1}^{0}+x_{2}\right)\left(x_{1}+x_{2}^{0}\right)} \\
H^{A}\left(x_{1}, x_{2}, x_{1}^{0}, x_{2}^{0}\right) & =-\frac{2}{x_{1} x_{2}\left(x_{1}+x_{2}\right)}
\end{aligned}
$$




$$
\begin{aligned}
G^{C}\left(x_{1}, x_{2}, x_{1}^{0}, x_{2}^{0}\right) & =\frac{2 x_{1}^{0} x_{2}^{0}-x_{1} x_{2}}{x_{1}^{2} x_{2}\left(x_{1}^{0}+x_{2}\right)} \\
H^{C}\left(x_{1}, x_{2}, x_{1}^{0}, x_{2}^{0}\right) & =\frac{x_{1}\left(x_{1}^{0}+x_{2}\right)\left(x_{2}-x_{2}^{0}\right)+2 x_{1}^{0} x_{2}^{0}\left(x_{1}+x_{2}\right)}{x_{1}^{2} x_{2}^{2}\left(x_{1}+x_{2}\right)^{2}} .
\end{aligned}
$$

Furthermore

$$
D_{g q}^{(1)}\left(x_{1}, x_{2}, x_{1}^{0}, x_{2}^{0}, \frac{M^{2}}{\mu_{F}^{2}}\right)=D_{q g}^{(1)}\left(x_{2}, x_{1}, x_{2}^{0}, x_{1}^{0}, \frac{M^{2}}{\mu_{F}^{2}}\right)
$$

with the factorization scale $\mu_{F}$, usually assumed to be given by $M$, and $x_{1,2}^{0}$ given by (3.4), with the constraints $\tau \leq x_{1,2}^{0} \leq 1$ and $-1+\tau \leq x_{F} \leq 1-\tau$. Alternatively one may define $x_{1,2}^{0}=\sqrt{\tau} e^{ \pm y}$ with the lepton pair rapidity $y$ in the hadron-hadron c.m. system being constrained by $\ln \sqrt{\tau} \leq y \leq-\ln \sqrt{\tau}$. The unpolarized cross sections for vector boson $\left(V B=W^{ \pm}, Z^{0}\right)$ production can be written as (for simplicity we use the same symbols for the coefficient functions as in (A.1) although they obviously differ for different processes)

$$
\begin{aligned}
\frac{\mathrm{d} \sigma_{h_{1} h_{2}}^{V B}\left(y, \mu_{F}^{2}\right)}{\mathrm{d} y}= & N^{V B} \sum_{q, q^{\prime}} c_{q q^{\prime}}^{V B} \int_{x_{1}^{0}}^{1} \mathrm{~d} x_{1} \int_{x_{2}^{0}}^{1} \mathrm{~d} x_{2} \\
\times & \left\{\left[D_{q \bar{q}}^{(0)}\left(x_{1}, x_{2}, x_{1}^{0}, x_{2}^{0}\right)+\frac{\alpha_{s}}{2 \pi} D_{q \bar{q}}^{(1)}\left(x_{1}, x_{2}, x_{1}^{0}, x_{2}^{0}, \frac{M_{V B}^{2}}{\mu_{F}^{2}}\right)\right]\right. \\
& \times\left\{q\left(x_{1}, \mu_{F}^{2}\right) \bar{q}^{\prime}\left(x_{2}, \mu_{F}^{2}\right)+\bar{q}\left(x_{1}, \mu_{F}^{2}\right) q^{\prime}\left(x_{2}, \mu_{F}^{2}\right)\right\} \\
+ & \frac{\alpha_{s}}{2 \pi} D_{g q}^{(1)}\left(x_{1}, x_{2}, x_{1}^{0}, x_{2}^{0}, \frac{M_{V B}^{2}}{\mu_{F}^{2}}\right) g\left(x_{1}, \mu_{F}^{2}\right)\left\{q^{\prime}\left(x_{2}, \mu_{F}^{2}\right)+\bar{q}^{\prime}\left(x_{2}, \mu_{F}^{2}\right)\right\} \\
+ & \left.\frac{\alpha_{s}}{2 \pi} D_{q g}^{(1)}\left(x_{1}, x_{2}, x_{1}^{0}, x_{2}^{0}, \frac{M_{V B}^{2}}{\mu_{F}^{2}}\right)\left\{q\left(x_{1}, \mu_{F}^{2}\right)+\bar{q}\left(x_{1}, \mu_{F}^{2}\right)\right\} g\left(x_{2}, \mu_{F}^{2}\right)\right\}
\end{aligned}
$$

with $N^{V B}=\sqrt{2} \pi G_{F} M_{V B}^{2} / 3 S$, using $M_{W}=80.42 \mathrm{GeV}$ and $M_{Z}=91.19 \mathrm{GeV}$, and $c_{q q^{\prime}}^{W^{ \pm}}=\left|V_{q q^{\prime}}\right|^{2}$ with the relevant CKM matrix elements $V_{u d} \approx 0.97$ and $V_{u s} \approx 0.22$, and 
$c_{q q^{\prime}}^{Z^{0}}=\delta_{q q^{\prime}}\left(v_{q}^{2}+a_{q}^{2}\right)$ with $v_{u}^{2}+a_{u}^{2} \approx 0.29$ and $v_{d}^{2}+a_{d}^{2} \approx 0.37$. Furthermore we have now $D_{q \bar{q}}^{(0)}\left(x_{1}, x_{2}, x_{1}^{0}, x_{2}^{0}\right)=\delta\left(x_{1}-x_{1}^{0}\right) \delta\left(x_{2}-x_{2}^{0}\right)$ and the $\mathrm{NLO}(\overline{\mathrm{MS}})$ coefficients $D_{q \bar{q}}^{(1)}$ and $D_{q g}^{(1)}$ are given by eqs. (15) and (16) of ref. [29], with $D_{g q}^{(1)}$ being again given by (A.5).

The doubly longitudinally polarized cross sections are generally defined via $\Delta \sigma_{\vec{h}_{1} \vec{h}_{2}}=$ $\frac{1}{4}\left(\sigma_{++}-\sigma_{+-}-\sigma_{-+}+\sigma_{--}\right)$. The relevant differential polarized Drell-Yan cross section for $\vec{h}_{1} \vec{h}_{2} \rightarrow \gamma^{*} X \rightarrow l^{+} l^{-} X$ is given by $\left(1,2\right.$ denote the arguments $x_{1,2}, \mu_{F}$ and $\ldots$ denotes the variables of the coefficient functions in (A.2-A.6))

$$
\begin{aligned}
M^{2} \frac{\mathrm{d} \Delta \sigma_{\vec{h}_{1} \vec{h}_{2}}^{\gamma_{2}}\left(x_{F}, M^{2}, \mu_{F}^{2}\right)}{\mathrm{d} M^{2} \mathrm{~d} x_{F}} & =-N^{\gamma^{*}} \sum_{q=u, d, s} e_{q}^{2} \int_{x_{1}^{0}}^{1} \mathrm{~d} x_{1} \int_{x_{2}^{0}}^{1} \mathrm{~d} x_{2} \\
\times & \left\{\left[D_{q \bar{q}}^{(0)}(\ldots)+\frac{\alpha_{s}}{2 \pi} D_{q \bar{q}}^{(1)}(\ldots)\right]\right. \\
& \times\{\Delta q(1) \Delta \bar{q}(2)+\Delta \bar{q}(1) \Delta q(2)\} \\
+ & \frac{\alpha_{s}}{2 \pi} \Delta D_{g q}^{(1)}(\ldots) \Delta g(1)\{\Delta q(2)+\Delta \bar{q}(2)\} \\
+ & \left.+\frac{\alpha_{s}}{2 \pi} \Delta D_{q g}^{(1)}(\ldots)\{\Delta q(1)+\Delta \bar{q}(1)\} \Delta g(2)\right\}
\end{aligned}
$$

where $D_{q \bar{q}}^{(0,1)}$ are as in (A.1) with $D_{q \bar{q}}^{(1)}$ given in (A.2) and [23]

$$
\begin{aligned}
& \Delta D_{q g}^{(1)}\left(x_{1}, x_{2}, x_{1}^{0}, x_{2}^{0}, \frac{M^{2}}{\mu_{F}^{2}}\right)=T_{F}\left\{\frac { \delta ( x _ { 2 } - x _ { 2 } ^ { 0 } ) } { ( x _ { 1 } ^ { 0 } + x _ { 2 } ^ { 0 } ) x _ { 1 } ^ { 2 } } \left[\left(2 x_{1}^{0}-x_{1}\right) \ln \frac{\left(x_{1}^{0}+x_{2}^{0}\right)\left(1-x_{2}^{0}\right)\left(x_{1}-x_{1}^{0}\right)}{x_{1}^{0} x_{2}^{0}\left(x_{1}+x_{2}^{0}\right)}\right.\right. \\
&+\left.2\left(x_{1}-x_{1}^{0}\right)\right]+\frac{\Delta G^{C}\left(x_{1}, x_{2}, x_{1}^{0}, x_{2}^{0}\right)}{\left(x_{2}-x_{2}^{0}\right)_{+}}+H^{C}\left(x_{1}, x_{2}, x_{1}^{0}, x_{2}^{0}\right) \\
&+\left.\ln \frac{M^{2}}{\mu_{F}^{2}}\left\{\frac{\delta\left(x_{2}-x_{2}^{0}\right)}{\left(x_{1}^{0}+x_{2}^{0}\right) x_{1}^{2}}\left(2 x_{1}^{0}-x_{1}\right)\right\}\right\}
\end{aligned}
$$


with $H^{C}(\ldots)$ given in (A.4) and

$$
\Delta G^{C}\left(x_{1}, x_{2}, x_{1}^{0}, x_{2}^{0}\right)=\frac{\left(x_{1}^{0} x_{2}^{0}\right)^{2}+\left(x_{1} x_{2}-x_{1}^{0} x_{2}^{0}\right)^{2}}{x_{1}^{3} x_{2}^{2}\left(x_{1}^{0}+x_{2}\right)} .
$$

Similarly to (A.5) we have

$$
\Delta D_{g q}^{(1)}\left(x_{1}, x_{2}, x_{1}^{0}, x_{2}^{0}, \frac{M^{2}}{\mu_{F}^{2}}\right)=\Delta D_{q g}^{(1)}\left(x_{2}, x_{1}, x_{2}^{0}, x_{1}^{0}, \frac{M^{2}}{\mu_{F}^{2}}\right) .
$$

The doubly longitudinally polarized cross sections for vector boson production are given by

$$
\begin{aligned}
\frac{\mathrm{d} \Delta \sigma_{\vec{h}_{1} \vec{h}_{2}}^{V B}\left(y, \mu_{F}^{2}\right)}{\mathrm{d} y}= & -N^{V B} \sum_{q, q^{\prime}} c_{q q^{\prime}}^{V B} \int_{x_{1}^{0}}^{1} \mathrm{~d} x_{1} \int_{x_{2}^{0}}^{1} \mathrm{~d} x_{2} \\
\times & \left\{\left[D_{q \bar{q}}^{(0)}(\ldots)+\frac{\alpha_{s}}{2 \pi} D_{q \bar{q}}^{(1)}(\ldots)\right]\right. \\
& \times\left\{\Delta q(1) \Delta \bar{q}^{\prime}(2)+\Delta \bar{q}(1) \Delta q^{\prime}(2)\right\} \\
+ & \frac{\alpha_{s}}{2 \pi} \Delta D_{g q}^{(1)}(\ldots) \Delta g(1)\left\{\Delta q^{\prime}(2)+\Delta \bar{q}^{\prime}(2)\right\} \\
+ & \left.\frac{\alpha_{s}}{2 \pi} \Delta D_{q g}^{(1)}(\ldots)\{\Delta q(1)+\Delta \bar{q}(1)\} \Delta g(2)\right\}
\end{aligned}
$$

with $N^{V B}, c_{q q^{\prime}}^{V B}$ and $D_{q \bar{q}}^{(0,1)}$ as in (A.6) and $\Delta D_{g q}^{(1)}$ is now given by eq. (18) of ref. [29] which relates to $\Delta D_{q g}^{(1)}$ again via (A.10).

Finally, the singly longitudinally polarized cross sections for vector boson $\left(V B=W^{ \pm}\right.$, $\left.Z^{0}\right)$ production are generally defined via $\Delta \sigma_{\vec{h}_{1} h_{2}}=\frac{1}{4}\left(\sigma_{++}+\sigma_{+-}-\sigma_{-+}-\sigma_{--}\right)$which are given by [29]

$$
\frac{\mathrm{d} \Delta \sigma_{\vec{h}_{1} h_{2}}^{V B}\left(y, \mu_{F}^{2}\right)}{\mathrm{d} y}=N^{V B} \sum_{q, q^{\prime}} c_{q q^{\prime}}^{V B} \int_{x_{1}^{0}}^{1} \mathrm{~d} x_{1} \int_{x_{2}^{0}}^{1} \mathrm{~d} x_{2}
$$




$$
\begin{gathered}
\times\left\{\left[D_{q \bar{q}}^{(0)}(\ldots)+\frac{\alpha_{s}}{2 \pi} D_{q \bar{q}}^{(1)}(\ldots)\right]\right. \\
\times\left\{-\Delta q(1) \bar{q}^{\prime}(2)+\Delta \bar{q}(1) q^{\prime}(2)\right\} \\
+\frac{\alpha_{s}}{2 \pi} \Delta D_{g q}^{(1)}(\ldots) \Delta g(1)\left\{q^{\prime}(2)-\bar{q}^{\prime}(2)\right\} \\
\left.+\frac{\alpha_{s}}{2 \pi} D_{q g}^{(1)}(\ldots)\{-\Delta q(1)+\Delta \bar{q}(1)\} g(2)\right\}
\end{gathered}
$$

where all normalizations, couplings and coefficient functions are as in (A.11) except for $c_{q q^{\prime}}^{Z^{0}}$ which is now given by $c_{q q^{\prime}}^{Z^{0}}=\delta_{q q^{\prime}} 2 v_{q} a_{q}$, i.e. $c_{u u}^{Z^{0}}=\frac{1}{2}-\frac{4}{3} \sin ^{2} \Theta_{W} \approx 0.19$ and $c_{d d}^{Z^{0}}=$ $c_{s s}^{Z^{0}}=\frac{1}{2}-\frac{2}{3} \sin ^{2} \Theta_{W} \approx 0.35$. 


\section{References}

[1] T. Morii and T. Yamanishi, Phys. Rev. D61(2000) 057501.

[2] B. Dressler, K. Goeke, M.V. Polyakov and C. Weiss, Eur. Phys. J. C14 (2000) 147.

[3] K. Ackerstaff et al., HERMES Collab., Phys. Lett. B464 (1999) 123.

[4] D. de Florian and R. Sassot, Phys. Rev. D62 (2000) 094025.

[5] S. Kumano and M. Miyama, Phys. Lett. B479 (2000) 149.

[6] B. Dressler et al., Univ. Bochum RUB-TPII-13/99 (hep-ph/9910464).

[7] D. Diakonov et al., Nucl. Phys. B480 (1996) 341; Phys. Rev. D56 (1997) 4096.

[8] K. Goeke et al., Univ. Bochum RUB-TPII-18/99 (hep-ph/0001272).

[9] M. Wakamatsu and T. Kubota, Phys. Rev. D60 (1999) 034020.

[10] R.S. Bhalerao, Phys. Lett. B380 (1996) 1; 387 (1996) 881 (E); talk given at the Int. Conf. on QNP 2000, Adelaide, Feb. 2000 (hep-ph/0003075);

R.S. Bhalerao, N.G. Kelkar and B. Ram, Phys. Lett. B476 (2000) 285.

[11] A.W. Thomas, Phys. Lett. 126B (1983) 97;

A.I. Signal and A.W. Thomas, Phys. Rev. D40 (1989) 2832;

E.M. Henley and G.A. Miller, Phys. Lett. B251 (1990) 453.

[12] P.V. Pobylitsa et al., Phys. Rev. D59 (1998) 034024; B. Dressler et al., Univ. Bochum RUB-TPII-15/98 (hep-ph/9809487).

[13] For recent reviews see, S. Kumano, Phys. Rep. 303 (1998) 183;

J. Speth and A.W. Thomas, Adv. Nucl. Phys. 24 (1998) 83.

[14] S.D. Ellis and W.J. Stirling, Phys. Lett. B256 (1991) 258. 
[15] A. Baldit et al., NA51 Collab. Phys. Lett. B332 (1994) 244;

E.A. Hawker et al., E866 Collab., Phys. Rev. Lett. 80 (1998) 3715;

J.C. Peng et al., E866 Collab., Phys. Rev. D58 (1998) 092004.

[16] R.D. Field and R.P. Feynman., Phys. Rev. D15 (1977) 2590.

[17] M. Glück and E. Reya, Mod. Phys. Lett. A15 (2000) 883.

[18] Y. Goto et al., AAC, Phys. Rev. D62 (2000) 034017.

[19] W. Vogelsang and M. Stratmann, private communication.

[20] M. Glück, E. Reya, M. Stratmann and W. Vogelsang, Phys. Rev. D53 (1996) 4775.

[21] M. Glück, E. Reya and A. Vogt, Eur. Phys. J. C5 (1998) 461.

[22] G. Bunce, N. Saito, J. Soffer and W. Vogelsang, RIKEN-BNL report thep ph/0007218);

S.E. Vigdor, Indiana Univ. report (hep-ex/9905034).

[23] T. Gehrmann, Nucl. Phys. B498 (1997) 245.

[24] J.M. Virey, Eur. Phys. J. C8 (1999) 283.

[25] C. Bourrely and J. Soffer, Phys. Lett. B314 (1993) 132.

[26] C. Bourrely and J. Soffer, Nucl. Phys. B423 (1994) 329.

[27] J. Soffer and J.M. Virey, Nucl. Phys. B509 (1998) 297.

[28] P.J. Rijken and W.L. van Neerven, Phys. Rev. D51 (1995) 44.

[29] T. Gehrmann, Nucl. Phys. B534 (1998) 21. 


\section{$\underline{\text { Figure Captions }}$}

Fig. 1. Polarized (anti)quark distributions in LO and NLO according to eqs. (2.2) - (2.6) in the flavor-broken sea scenario (full curves) at various fixed factorization scales $\mu_{F}^{2} \equiv$ $Q^{2}$. For comparison the corresponding 'standard' flavor-symmetric sea distribution $\Delta \bar{q}$ according to AAC [18] is shown by the dashed curves. The remaining $\Delta u$ and $\Delta d$ densities are similar in both scenarios (cf. fig. 5).

Fig. 2. The unpolarized Drell-Yan dilepton production cross section $d^{2} \sigma_{p p}^{\gamma^{*}(M)} / d M d x_{F}$ for $\sqrt{S}=50$ and $100 \mathrm{GeV}$ in NLO based on the GRV98 21] NLO parton distributions.

Fig. 3. The ratio $R_{p+n}$ of polarized $\vec{p} \vec{p}$ and $\vec{p} \vec{n}$ Drell-Yan cross sections in eq. (3.3) and the asymmetry $A_{\vec{p}-\vec{n}}^{\gamma^{*}(M)}$ in eq. (3.2) in LO and NLO based on the distributions of the broken and unbroken (AAC) sea scenarios as specified in fig. 1. The perturbative stability of the predicted asymmetries and their sensitivity to the choice of the factorization scale $\mu_{F}$ is examined in the two lower figures. Statistical errors are calculated according to eqs. (3.5) and (3.6). The 'crude approx.' curve refers to $[1+\Delta \bar{d}(2) / \Delta \bar{u}(2)] / 2$ in eq. (3.3).

Fig. 4. The unpolarized differential cross sections $d \sigma_{p p}^{W^{ \pm}, Z^{0}} / d y$ for $\sqrt{S}=500 \mathrm{GeV}$ (RHIC) in NLO based on the GRV98 [21] NLO parton distributions.

Fig. 5. The LO (anti)quark asymmetries $(\Delta \bar{q} / \bar{q}) \Delta q / q$ at the relevant factorization scale $\mu_{F}^{2} \equiv Q^{2}=M_{W}^{2}$, dominating the single spin asymmetries for $A_{\vec{p} p}^{W^{ \pm}}$at $|y| \gtrsim \frac{1}{2}$ according to eqs. (3.8) and (3.9).

Fig. 6. The double spin asymmetries $A_{\vec{p} \vec{p}}^{W^{+}}\left[\right.$eq. (3.10)] and $A_{\vec{p} \vec{p}}^{W^{-}}$[eq. (3.11)] at $\sqrt{S}=500$ $\mathrm{GeV}$ in the broken and unbroken (AAC) sea scenarios. The statistical errors are evaluated according to eq. (3.5). The perturbative stability of the predicted asymmetries and their sensitivity to the choice of the factorization scale $\mu_{F}$ is shown as well. 
Fig. 7. The single spin asymmetries $A_{\vec{p} p}^{W^{+}}$[eq. (3.8)] and $A_{\vec{p} p}^{W^{-}}$[eq. (3.9)] at $\sqrt{S}=500 \mathrm{GeV}$ in the broken and unbroken (AAC) sea scenarios, as specified in fig. 1. The statistical errors are evaluated according to eq. (3.7). The errors on the AAC curves are similarly small as in the broken scenario. The quality of the 'crude' approximations in eq. (3.8), $\Delta \bar{d}(1) / \bar{d}(1)$, and eq. (3.9), $\Delta \bar{u}(1) / \bar{u}(1)$, is examined as well. The perturbative stability of the predicted asymmetries is similar to the one shown in fig. 6.

Fig. 8. The ratios $\Delta \sigma_{\vec{p} \vec{p}}^{W^{+}} / \Delta \sigma_{\vec{p} \vec{p}}^{W^{-}}$and $\Delta \sigma_{\vec{p} d}^{W^{+}} / \Delta \sigma_{\vec{p} d}^{W^{-}}$for doubly and singly polarized cross sections, respectively, at $\sqrt{S}=500 \mathrm{GeV}$ obtained via eqs. (3.15) and (3.14). The meaning of the curves corresponds to that in fig. 7 .

Fig. 9. The asymmetries $a_{\vec{p} \vec{N}}^{W}$ and $a_{\vec{p} N}^{W}$ at $\sqrt{S}=500 \mathrm{GeV}$ in eqs. (3.18) and (3.17), respectively, for the broken and unbroken (AAC) scenario.

Fig. 10. The asymmetries $A_{\vec{p} \vec{p}}^{Z^{0}}$ and $A_{\vec{p}, p-n}^{Z^{0}}$ in LO and NLO at $\sqrt{S}=500 \mathrm{GeV}$ given in eqs. (3.19) and (3.20), respectively. The meaning of the curves corresponds to that in fig. 9 . 



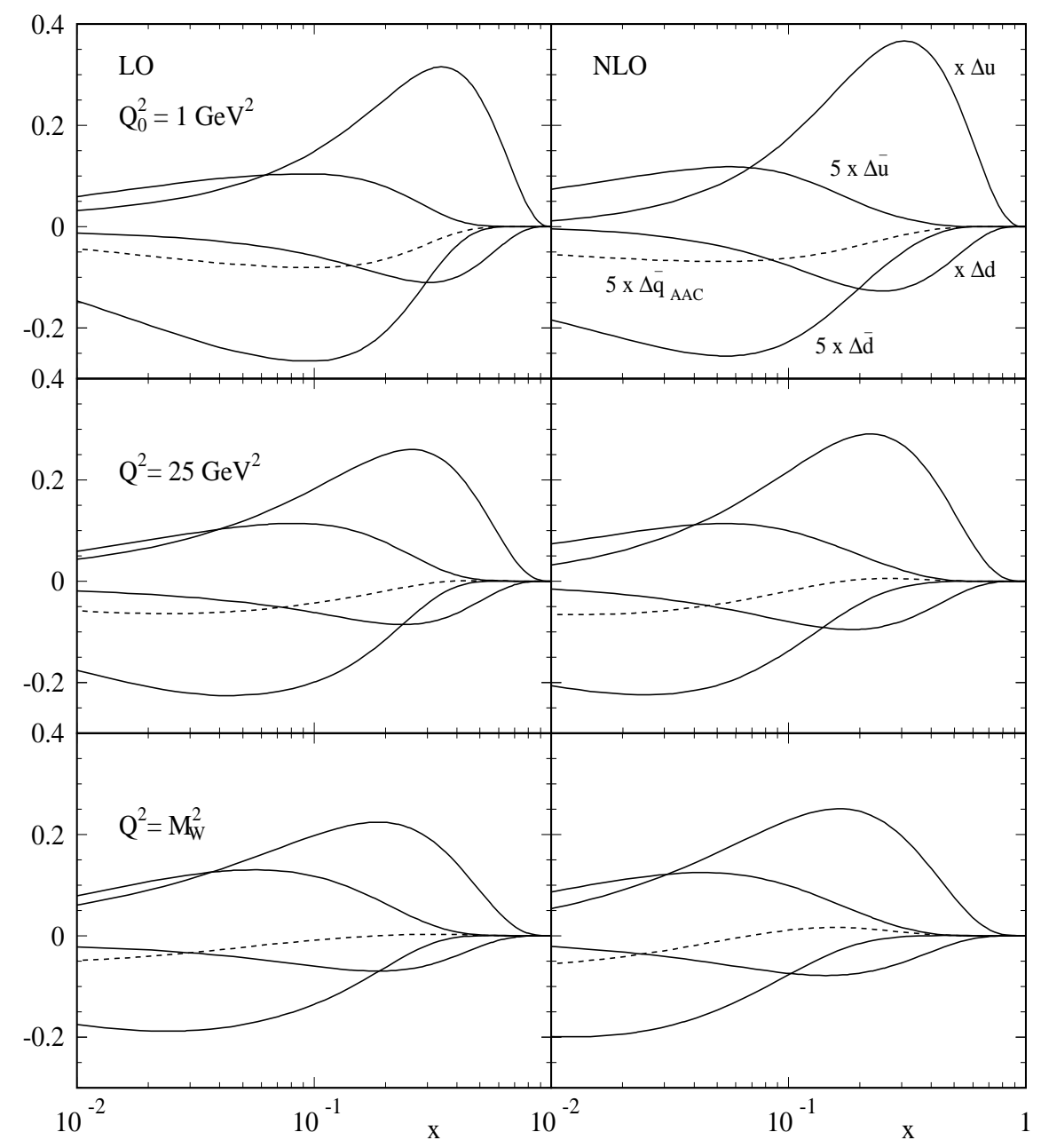

Figure 1 


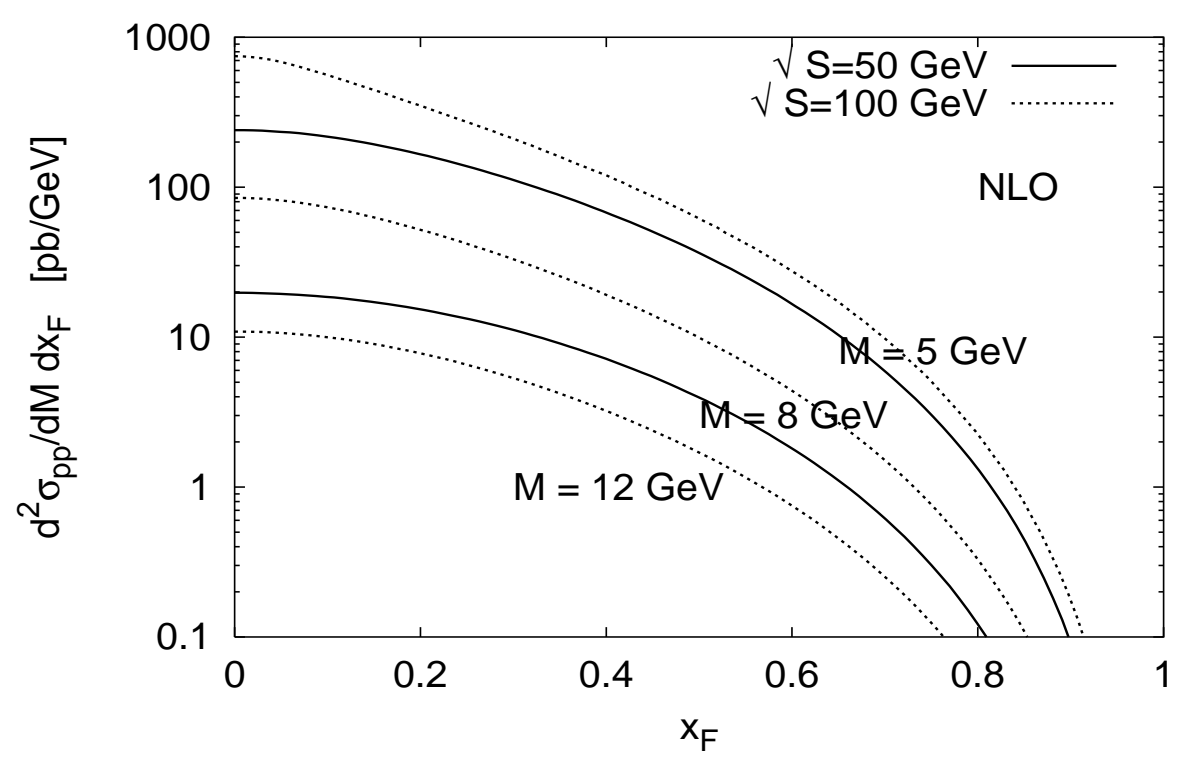

Figure 2
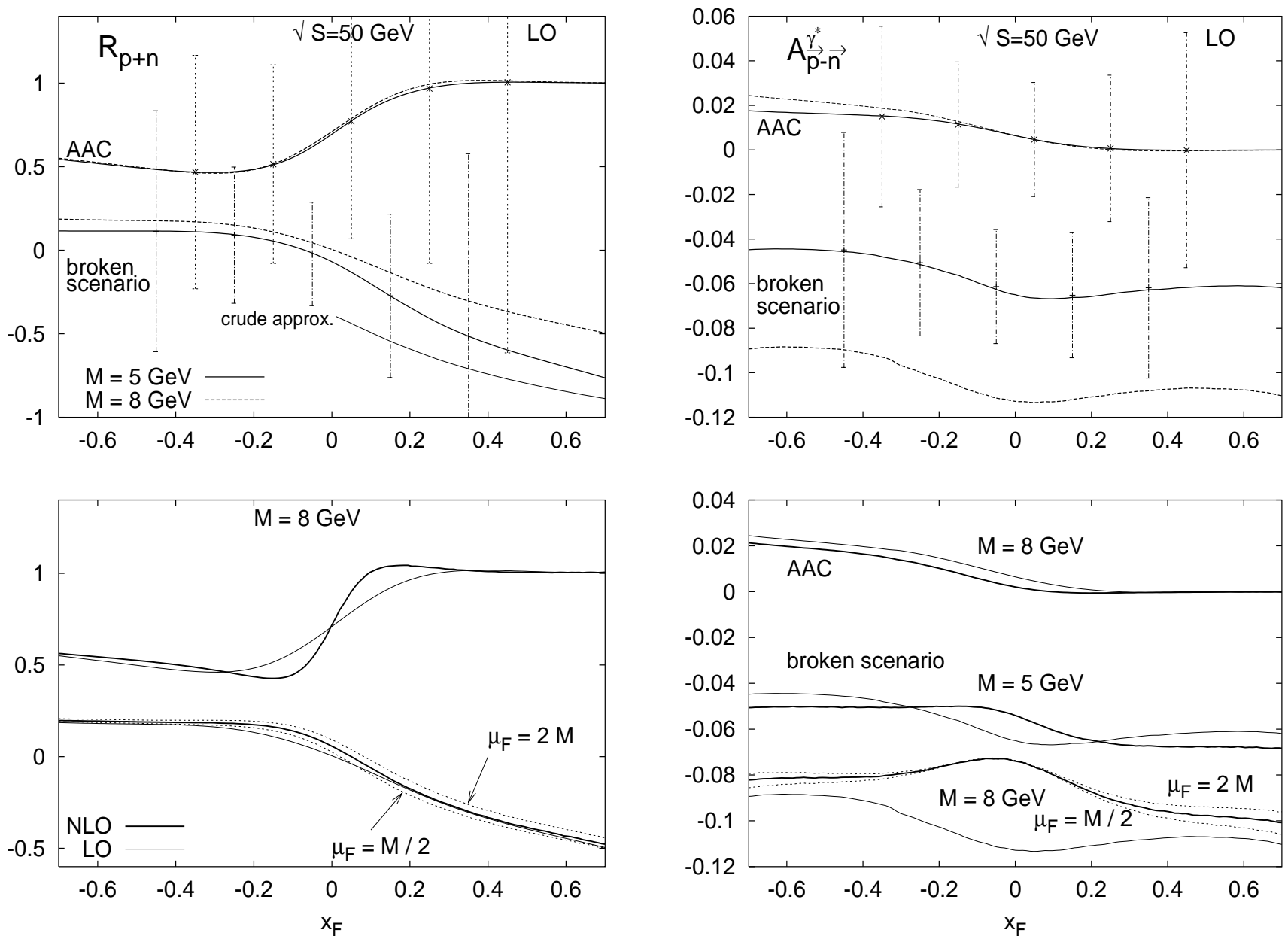

Figure 3 


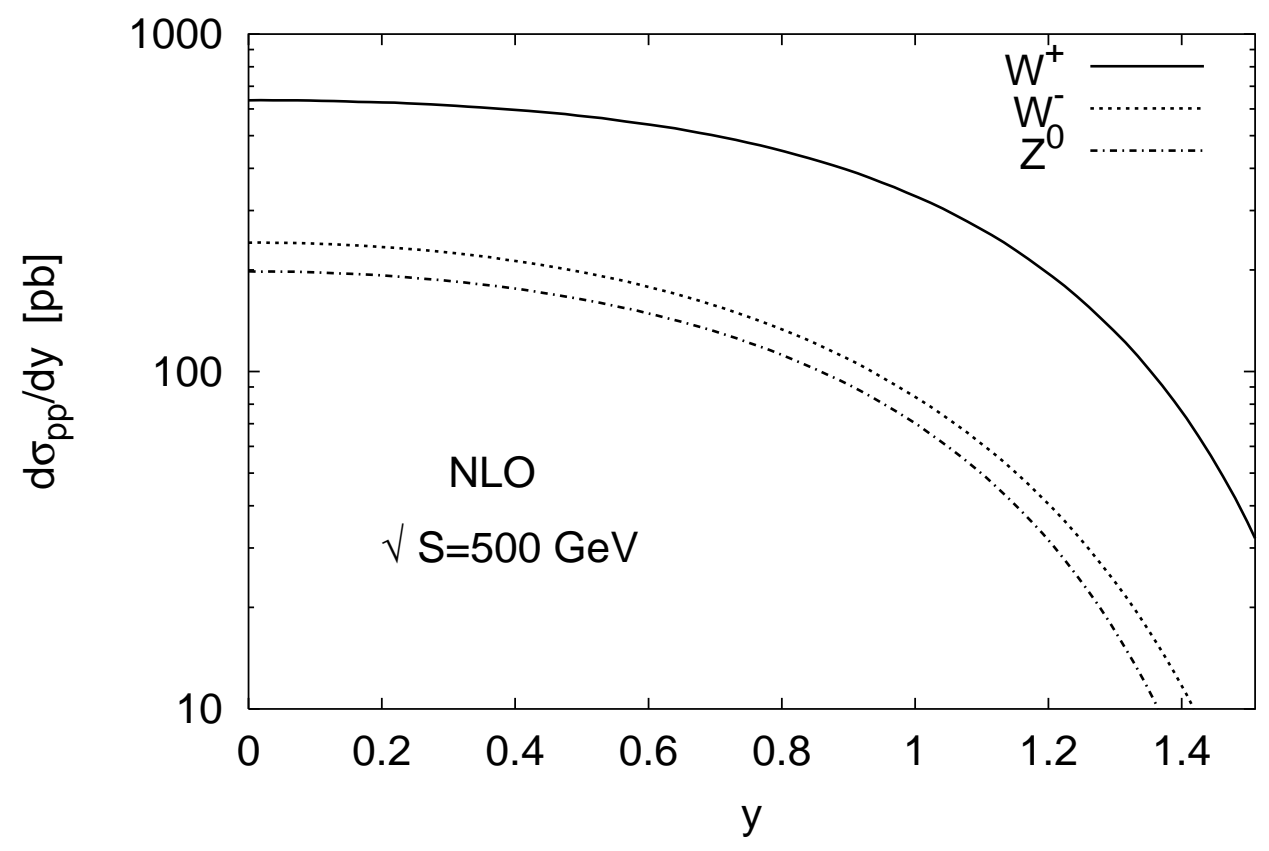

Figure 4

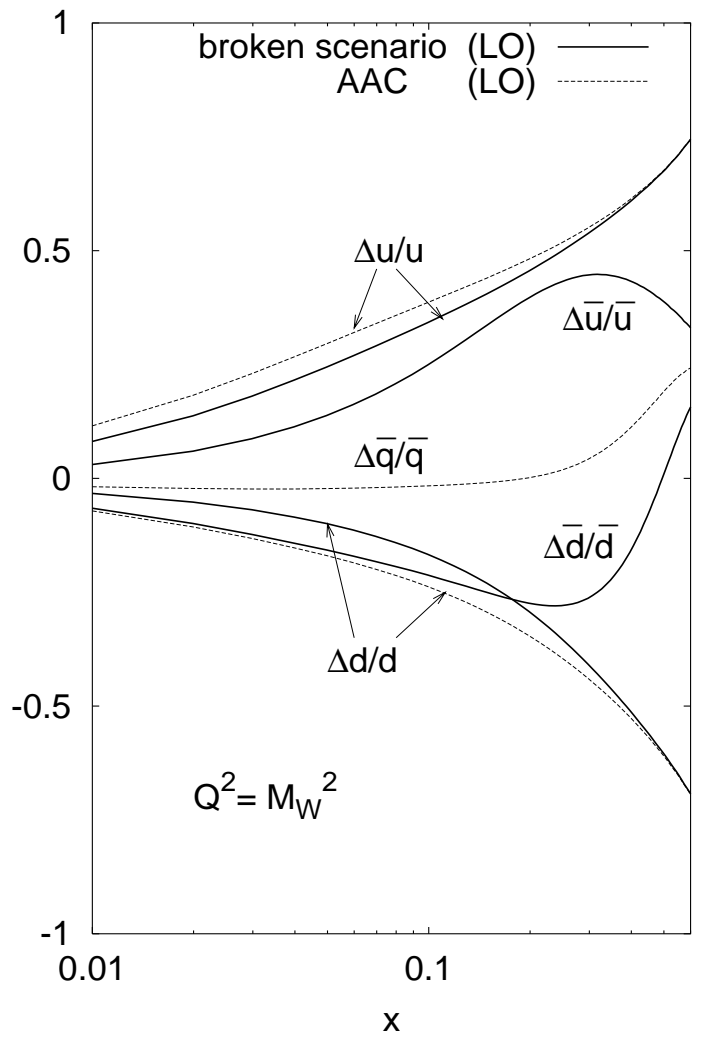

Figure 5 

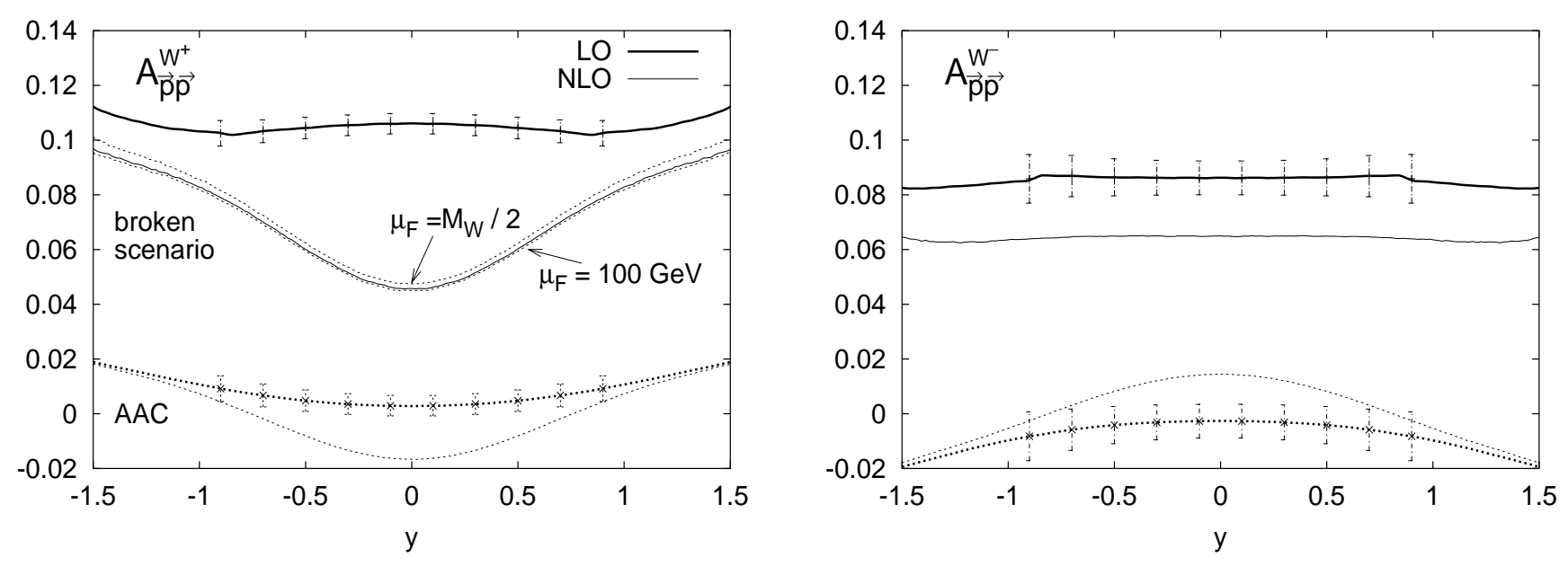

Figure 6
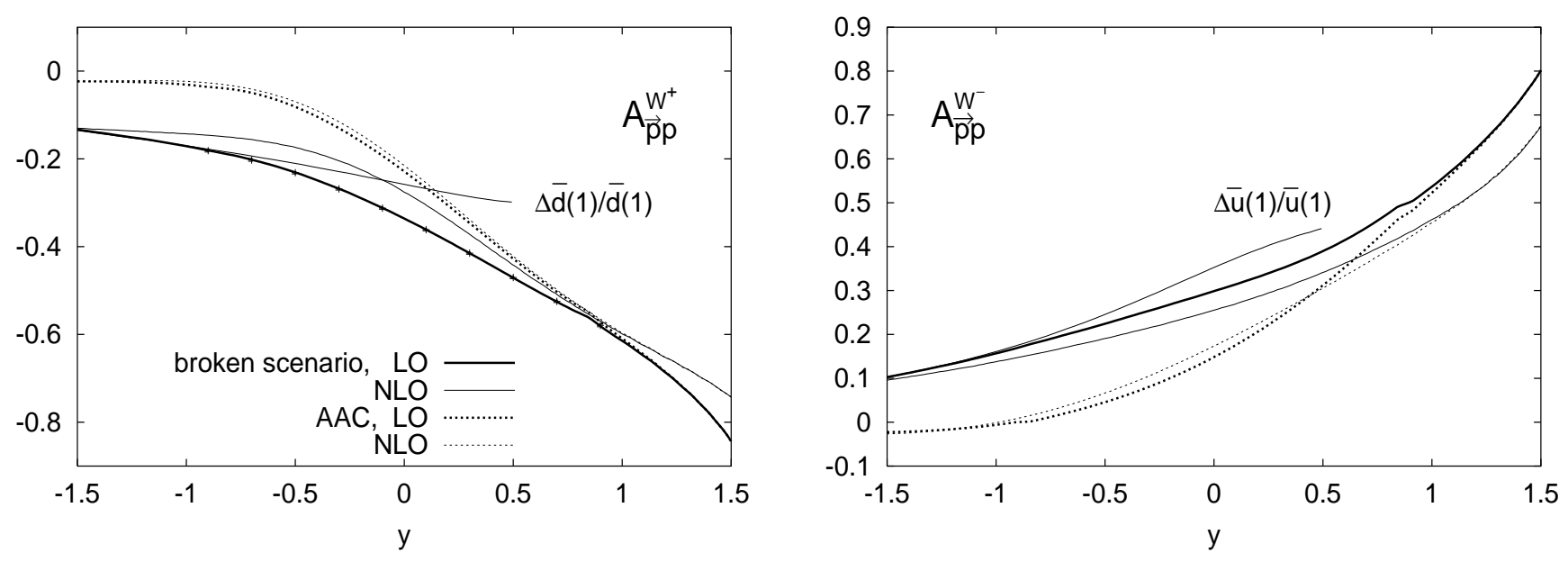

Figure 7 

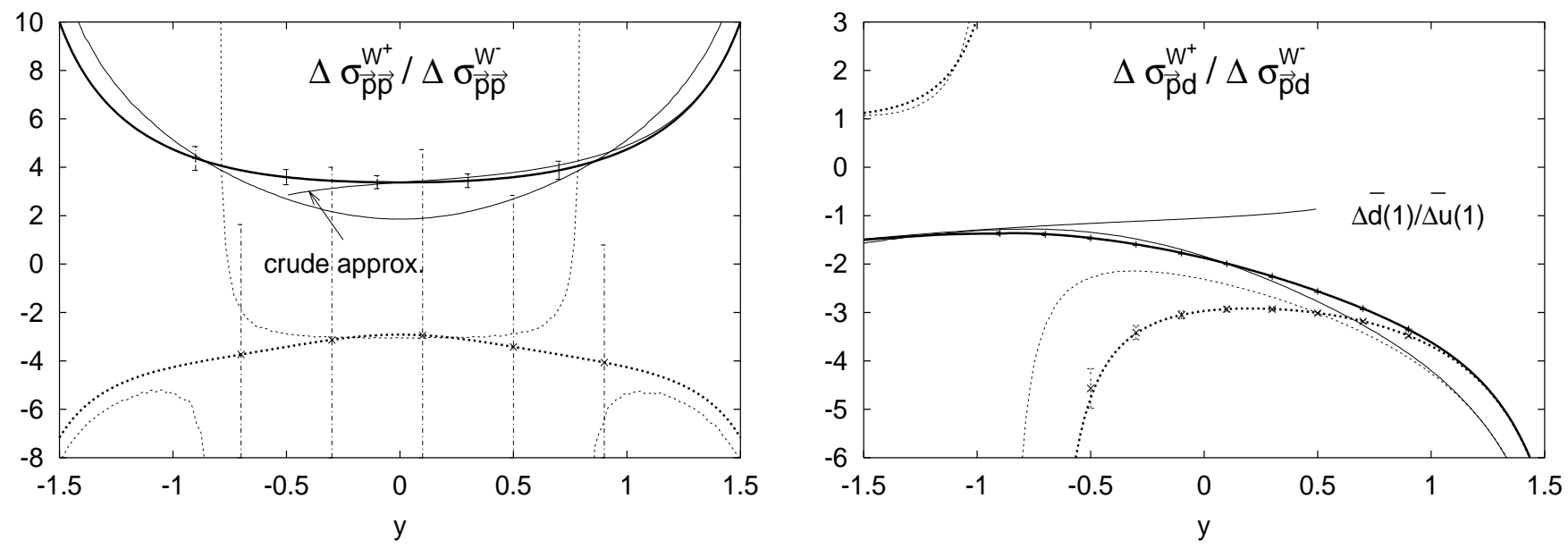

Figure 8
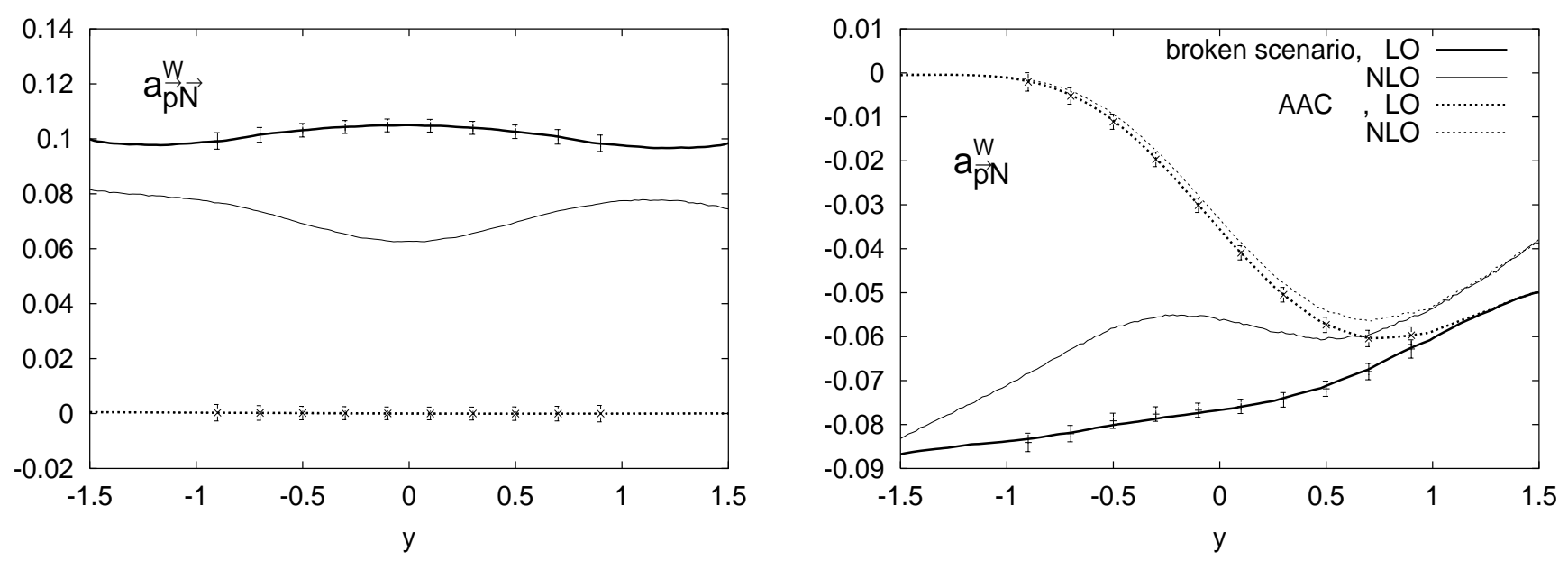

Figure 9
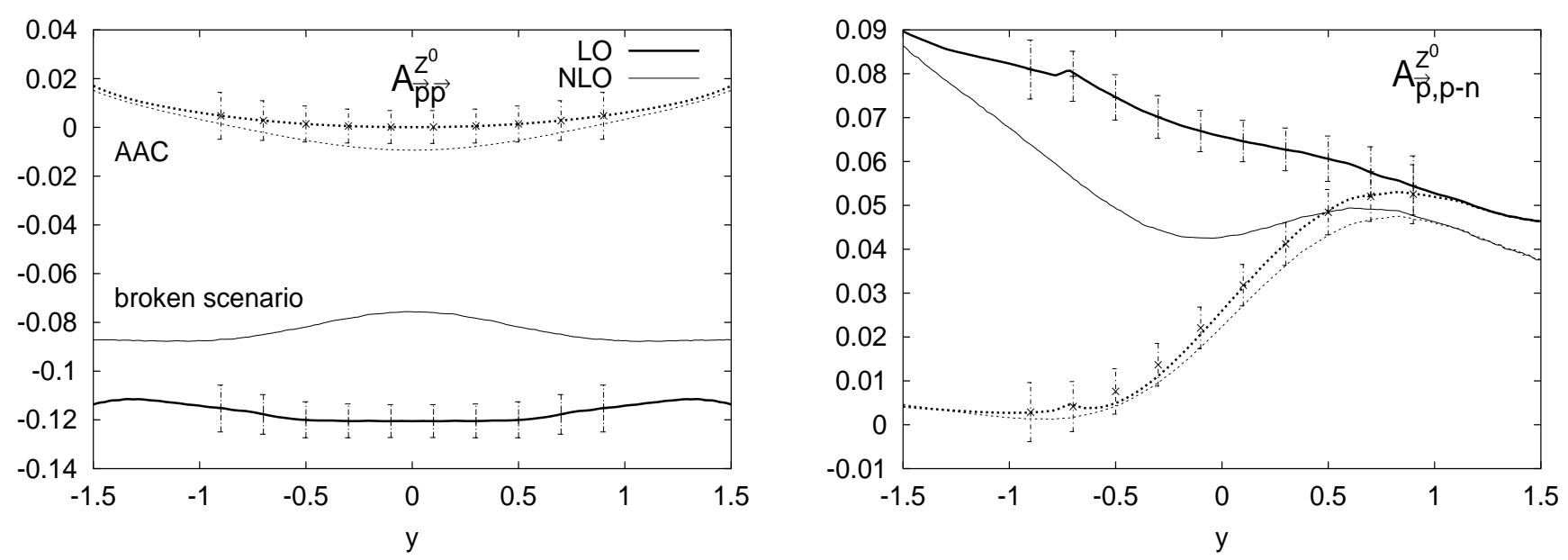

Figure 10 